\title{
Drying Process Simulation Methodology based on Chemical Kinetics Laws
}

\author{
Vladimir M. Arapov ${ }^{1}$, Dmitriy A. Kazartsev ${ }^{2}$, Igor A. Nikitin ${ }^{3}$, Maria V. Babaeva ${ }^{4}$, Svetlana V. Zhukovskaya ${ }^{5}$ \\ Svetlana N. Tefikova ${ }^{6}$, Galina V. Posnova ${ }^{7}$, Igor V. Zavalishin ${ }^{8}$ \\ Voronezh State University of Engineering Technologies, Voronezh, Russian Federation ${ }^{1}$ \\ K.G. Razumovsky Moscow State University of Technologies and Management \\ (The First Cossack University) Moscow, Russian Federation, 2, 4, 5, 6, 7, 8
}

\begin{abstract}
It is shown that the existing approaches to drying process modeling, based on a system of interconnected differential equations of heat and mass transfer or on statistical processing of drying process experimental data, have significant drawbacks. It greatly complicates the development of computer means for controlling production processes. During modeling it is proposed to consider drying process from the standpoint of physical chemistry as a quasi-topochemical heterogeneous reaction and perform mathematical modeling of this process based on the laws of chemical kinetics. The basic issues of methodology of drying process modeling based on the laws of chemical kinetics are reviewed: the study of the equation of drying rate during the removal of free and bound moisture; methods for determining composition of the aqueous fractions with different forms and energy of moisture in materials; methods of determination of an activation energy of moisture; the influence of the concentration of moisture and other process factors on the drying speed. The methodological approach considered in the article allows developing reliable mathematical models of drying kinetics for the purposes of computer technologies for managing production processes and avoiding the errors that the authors note in previously published works.
\end{abstract} energy

Keywords-Modeling; drying; chemical kinetics; activation

\section{INTRODUCTION}

The current stage of industrial development is characterized by the transition to computer technology for managing production processes. The pace of this development largely depends on the level of mathematical modeling of technological processes - the completeness and accuracy of reflection by a mathematical model of all the phenomena accompanying the technological process. Computer technology allows not only to increase the culture of production and labor productivity, but also to optimize energy and material costs for production $[1,2]$. Therefore, the development of reliable mathematical models of modern technological processes is an urgent task for many industries. In particular, this problem is relevant for the drying process.

When modeling drying process, it is necessary to consider a number of complex interrelated phenomena [3]. The diversity of these phenomena and their interrelation cause the process to be considered from different points of view. From the standpoint of the classical theory of drying, this process represents a set of simultaneously occurring phenomena of heat transfer and mass transfer $[4,5,6]$ : the transfer of heat from gas to the material through a boundary layer of gas; the evaporation of moisture from the surface of the material; the movement of moisture vapor from the material surface in the environment of the drying agent through the boundary layer of gas; transfer of heat and moisture inside the material.

Most products consist of three phases: solid, liquid, and gaseous. The mass of the gaseous phase is very small. Therefore, it is believed that a wet body consists of absolutely dry material and moisture. When interacting with the solid phase, moisture molecules form a bond of a certain strength. The drying process is accompanied by a violation of this connection, which requires a certain amount of energy. The binding energy of moisture to the material depends on the nature of the formation of bond forms, which may have a chemical, physical-chemical or physical-mechanical origin. The connection of moisture with the material is significantly affected by the phenomenon of adsorption. The mechanism of physical adsorption is very complex. Different areas of the surface have different adsorption potentials, and there is no strictly justified complete theory of this phenomenon, which significantly complicates the analytical determination of the energy of the connection of moisture with the material and mathematical modeling of drying.

The mechanism of moisture transfer in capillary-porous bodies, which many products belong to, is very complex and includes the phenomena of convective filtration transfer in macro-and micro-capillaries, the phenomena of diffusion, effusion, thermodiffusion, and others [5]. But even in macrocapillaries, the molar and diffusive transfer of moisture is of different nature, so mathematical modeling of the total flow of moisture, taking into account the totality of all phenomena, is a difficult task.

In the process of thermal drying a gradient of temperature and moisture content occurs in the material. This causes the movement of moisture in the material both due to the temperature gradient (the phenomenon of thermal conductivity or thermal diffusion) and due to the humidity gradient (the phenomenon of moisture conductivity or concentration diffusion) [3, 4]. Due to the humidity gradient, moisture moves to the evaporation surface, and under the influence of the temperature gradient, it can move from the surface layers to the inside of the material. Thermal and moisture conductivity is a complex physical phenomenon that consists of a number of processes and its mathematical description requires a large 
amount of information about the properties of the product as an object of drying. Thus, the phenomena of mass transfer during heat drying are not only complex in their thematic description, but also have a contradictory character.

Heat transfer and mass transfer phenomena are no less complex during heat drying $[3,4,5,6]$. Thus, in general, it is not possible to obtain an accurate analytical solution for calculating heat transfer and mass transfer of heat drying, since for a mathematical description of all phenomena, it is necessary to know a large amount of information about the properties and parameters of the drying agent and product, which may not change deterministically.

Possible approaches to modeling drying are analyzed below. A relatively new approach to modeling based on the laws of chemical kinetics is considered. A critical analysis of published works in this area is given, and the methodology for modeling drying based on the laws of chemical kinetics is briefly described.

\section{ANALYTICAL REVIEW}

Over the past decades scientific provisions on drying have been continuously developed. A large number of mathematical models have been published for various drying methods, most of which are based either on a system of differential interrelated equations of mass and energy transfer [6, 13], or on statistical processing of experimental research data for a specific product $[3,7,8,9,10,11,12]$.

Sufficient information about the main directions of this development can be found at some practical conferences [6]. Modern mathematical models of drying are computer models, since their application to calculation and management of the process is carried out using a computer due to the complexity. However despite the use of computer technology, models in the form of a system of differential equations are difficult for practical use since they contain a large number of coefficients and characteristics of the properties of the processed product, these coefficients for each product and drying mode are often impossible to predict [3, 4]. The value of the coefficients depends on many random factors in preceding drying production stages. All this significantly reduces the reliability of these models. The development of mathematical modeling of drying processes with these approaches is hindered by a lack of knowledge about the mechanism of interaction of phases, as well as about the properties of the product that change during the drying process. Further development of drying modeling, as the author rightly notes [13], is possible on the application of achievements in the field of physical chemistry.

As is known from the theory of similarity and modeling, the application of models based on experimental studies is limited by the range of the study. The accuracy and reliability of these models is not high. These circumstances determine the relevance of the search for new approaches to drying process modeling and obtaining more reliable mathematical models of process kinetics.

\section{PhysicochemicAl APPROACH to Drying PROCESS MODELING}

From our point of view very promising in this direction is a physicochemical approach based on the idea of drying as a quasi-topochemical heterogeneous reaction. Here a dry residue and a vaporous phase, that has transferred to a drying agent, appear from the initial product as a result of physicochemical and phase transformations. Such a representation of drying allows applying the laws of kinetics of topochemical reactions of heterogeneous processes to mathematical modeling. First of all, two fundamental provisions of formal chemical kinetics: the law of acting masses and the Arrhenius kinetic equation are considered [14].

The law of acting masses is based on the assumption that the reaction rate depends on the number of collisions of reacting molecules, which is proportional to the product of the concentrations of reacting substances in degrees, whose indices correspond to stoichiometric coefficients of the chemical reaction equation.

The Arrhenius equation allows us to determine the value of the reaction rate constant $\mathrm{k}$, and in practical application it usually has the form:

$k=A \exp \left(-\frac{E}{R T}\right)$,

where $A$ is the preexponential factor, depending on the physicochemical properties of the reaction system, $E$ is the activation energy, $T$ is the absolute reaction temperature.

One of the first such works [15] some advantages of the physicochemical approach to drying are shown. The main advantage of this approach is to establish an explicit form of the drying rate equation, which does not require complex mathematical calculations for practical use in engineering calculations. Further studies $[16,17,18]$ confirmed the prospects of this approach to mathematical modeling of drying. An indisputable advantage of this approach is the possibility of using modern high-precision thermal analysis instruments for the numerical determination of process characteristics that are part of the mathematical model of the drying process, for example, the $E$ value.

However, in some recent scientific papers [19, 20, 21, 22] based on the physicochemical approach to the kinetics of drying process modeling, some research results, in our opinion, significantly contradict the generally accepted provisions of the theory of drying. So in [19], the author distinguishes two stages of the process of free water removal during wood drying. In this case, the activation energy of free water molecules at individual stages differs almost twofold at a sample temperature of $295 \ldots 296 \mathrm{~K}$, the E/R value is $55932 \ldots 32608$ $\mathrm{K}$, which corresponds to an activation energy of $465019 \ldots$ $271103 \mathrm{~kJ} / \mathrm{kmol}$, and at a temperature of $303 \ldots 298 \mathrm{~K}$, the E/R value is $34060 \ldots 62035 \mathrm{~K}$, which corresponds to an activation energy of $283175 \ldots 515759 \mathrm{~kJ} / \mathrm{kmol}$. In addition, the indicated values of the activation energy exceed the enthalpy of saturated water vapor at given temperatures by an order of magnitude (the enthalpy of saturated water vapor at a temperature of $303.15 \mathrm{~K}$ is $46008 \mathrm{~kJ} / \mathrm{kmol}$ ), which cannot but raise doubts about the reliability of the author's results [19]. And according 
to [10], the activation energy for drying mushrooms in the temperature range $65 \ldots 85^{\circ} \mathrm{C}$ is $22 \mathrm{~kJ} / \mathrm{mol}$. These facts indicate that there is no scientifically based method for determining the activation energy of water in relation to drying processes.

In $[19,20]$ the question of the influence of relative air humidity on the value of activation energy is considered. But the relative humidity of the air, as is known [3], has an effect on the external heat and mass transfer, when water molecules have already passed into a vaporous state. It is no coincidence that in the graphs of the dependence of the activation energy on the relative air humidity $[19,20]$ it is difficult to distinguish the indicated functional dependence due to the significant scatter of the experimental data, and the correlation estimate is not given to the corresponding equations.

A number of authors believe that the destruction of the moisture bond with the dry part of the product occurs in "their (different) temperature ranges" [19]. This means that the removal of water fractions with different binding energies during drying should occur in a strictly defined temperature range. But from the practice of drying, it is known that bound moisture is removed from the product even at relatively low temperatures, if the air is dried. We believe that the contradictory information available in the scientific literature on the issue under consideration indicates the absence of a reliable methodological approach to the application of laws of chemical kinetics to the analysis and modeling of drying processes. In this regard, the goal of this work is to substantiate the main approaches to drying processes modeling based on the laws of chemical kinetics. The object of research is the kinetics of products thermal drying. The subject of the study is the methodology of applying the laws of chemical kinetics to the modeling of drying processes.

\section{Methodology of a PhysicochemicAl APPROACH To DRYING PROCESS MODELING}

First of all, in the general case, it should be considered that the moisture in the material has a many fractional composition. Water fractions are distinguished by the binding energy to the dry part of the product. To determine the boundaries of each water fraction, appropriate studies should be carried out. The most complete and reliable information on the fractional composition of water in materials is provided by methods based on nuclear magnetic resonance [23] and thermal analysis methods $[14,24,25]$. A quantitative assessment of the bond strength of moisture with the dry part of the product can be obtained by the method of [26].

The drying rate of each water fraction based on the law of masses and the Arrhenius equation can be represented as:

$\frac{d \alpha_{i}}{d \tau}=f_{i}\left(\alpha_{i}\right) A_{i} \exp \left(-\frac{E_{i}}{R T}\right)$

where $\alpha_{i}=\frac{U_{i}^{\mathrm{H}}-U_{i}}{U_{i}^{\mathrm{H}}-U_{i}^{p}}-$ the degree of conversion of the $i$-th aqueous fraction;

$f_{i}\left(\alpha_{i}\right)$ - function of the degree of conversion of the $i$-th aqueous fraction;

$A_{i}$ - preexponential factor of the $i$-th water fraction, $\sec ^{-1}$;

\section{$\mathrm{K})$;}

$E_{i}$ - activation energy of the $i$-th aqueous fraction, $\mathrm{J} /(\mathrm{mol}$

$\mathrm{T}$-the absolute temperature of the $i$-th aqueous fraction equal to the temperature of the material, $\mathrm{K}$.

$U_{i}^{\mathrm{H}}, U_{i}^{p}, U_{i}$-the moisture content of the material, calculated as the ratio of the amount of the $i$-th aqueous fraction to the amount of the dry part of the product, respectively, the initial state, equilibrium state and the state considered at a given moment in time.

In detailing equation (2), it is necessary to determine the sequence of removal of water fractions based on information on the forms of moisture bonding in a material. Can the removal of water fractions be considered as parallel independent chemical reactions, or as sequential reactions, or is there some other order possible? If water clusters with different binding energies can be in different product areas, then the option of parallel removal of water fractions is possible. In this case, the equation for the drying rate will be:

$\frac{d \propto}{d \tau}=\sum f_{i}\left(\alpha_{i}\right) A_{i} \exp \left(-\frac{E_{i}}{R T}\right)$

If moisture is retained layer by layer by adsorption forces (moisture of monoadsorption and polyadsorption layers) and differs significantly in different layers in terms of activation energy, then at moderate drying temperatures, a model of successive removal of fractions is likely. If there is a film of free moisture on the surface of the plant cell, then the simultaneous removal of film and intracellular moisture is unlikely. Deletion of these fractions should be considered sequentially. In this case, the drying rate will be determined by the removal rate of the next aqueous fraction currently being considered:

$\frac{d \propto}{d \tau}=f_{i}\left(\alpha_{i}\right) A_{i} \exp \left(-\frac{E_{i}}{R T}\right)$

More complex options are also possible. For example, when drying casein [15], protein denaturation and the transition of bound moisture to a free state are possible.

Difficulties arise in determining the explicit form of the function of the degree of transformation $f_{i}\left(\alpha_{i}\right)$. For this, it is necessary to compare the studied drying method with the already known topochemical reactions [14] and select the closest option. Otherwise, you can take the option that is often encountered in practice:

$f_{i}\left(\alpha_{i}\right)=\left(1-\alpha_{i}\right)^{n}$,

where $n$ is a value showing the order of the reaction.

When choosing the type $f_{i}\left(\alpha_{i}\right)$ for free water, it is necessary to take into account the position known in the theory of drying: with constant heat supply to the material, the rate of free water removal is constant and does not depend on moisture content [3]. Therefore, for this fraction, the reaction order should be taken equal to zero. In addition, during this drying period, the temperature of the product is comparable to the temperature of the wet thermometer under these drying conditions [3]. Therefore, when free water is removed, the mathematical model of the drying rate has the form: 
$\frac{d \alpha}{d \tau}=A_{\mathrm{cB}} \exp \left(-\frac{E}{R T_{\mathrm{M}}}\right)$

where $A_{\mathrm{CB}}$ - preexponential factor when removing free moisture, $\sec ^{-1}$;

$T_{\mathrm{M}}$ - absolute temperature of the drying agent by wet thermometer, $\mathrm{K}$.

If we assume that for the transition of a free water molecule to a vapor state, additional energy is required equal to only the latent heat of vaporization $r$, then in this case we can take $E=r$.

Unfortunately, this was not taken into account in [19].

In our opinion, the order of magnitude of the activation energy can be estimated theoretically. Firstly, for a water molecule to pass from a liquid to a vapor state, it is necessary to break its connection with the dry part of the product, i.e. to give additional energy to the water molecule equal to (and possibly somewhat larger) the binding energy of moisture with the material $E_{i}^{\mathrm{cB}}$. For the transition of the molecule into a vaporous state, additional energy is required equal to the latent heat of vaporization. If the generated water vapor is not removed from the external surface of the product, but from the deepened evaporation zone [3], then additional energy will be needed to move the vapor through the dry layer of the product. During this movement, the initially formed saturated steam may overheat. Thus, the activation energy of the $i$-th aqueous fraction can be estimated as:

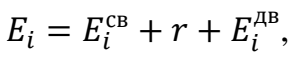

where $E_{i}$ - activation energy of the $i$-th aqueous fraction, $\mathrm{J} / \mathrm{mol}$;

$E_{i}^{\mathrm{cB}}-$ binding energy of the $i$-th moisture fraction with the material, J/mol;

$r$-latent heat of vaporization, $\mathrm{J} / \mathrm{mol}$;

$E_{i}^{\text {дв }}$ - energy required to move moisture to the outer surface of the product, $\mathrm{J} / \mathrm{mol}$.

Due to the fact that the heat of vaporization decreases with increasing temperature, then for $r \gg E_{i}^{\mathrm{cB}} \mathrm{u} r \gg E_{i}^{\mathrm{AB}}$, a decrease in the activation energy of the i-fraction is resulted with an increase in the temperature of the product and, as a consequence of this, an increase of the drying rate.

P.A. Rebinder proposed to determine the binding energy by the equation $E_{\mathrm{CB}}$.

$E_{\mathrm{cB}}=-R T \ln [\varphi(U, T)]$,

where $\varphi(U, T)$ - the relative humidity of the air in equilibrium with the material having a moisture content of $U$ and an absolute temperature of $T$;

$T$ - absolute temperature of the product, $\mathrm{K}$;

$R$ - universal gas constant, $\mathrm{J} /(\mathrm{mol} \cdot \mathrm{K})$.

Equation (8) characterizes the binding energy as the free energy of the isothermal separation of one mole of water from the dry part of the product without changing the state of aggregation [27]. It shows that $E_{\mathrm{CB}}$ is a function of the moisture content and temperature of the product, and not the relative humidity of the air supplied to the dryer, since each value of the moisture content of the product at temperature $T$ corresponds to a strictly defined value of the relative humidity of the air in the equilibrium system: air - product.

However, drying in an industrial apparatus is an irreversible and nonequilibrium process. Therefore, the actual energy consumption for breaking the bond of moisture with the material will probably be greater than the value calculated by equation (8). The value of $E_{i}^{\text {ДВ }}$ can be approximately determined as the energy cost of overcoming the hydraulic resistance during the movement of water vapor through capillaries of the dry layer of the product.

We believe that in order to establish the explicit form of mathematical models (4), (5), (6), the value of the activation energy should be determined experimentally on the basis of existing $[14,24]$ or the development of new thermal analysis methods.

The most difficult task is to determine the value of the preexponential factor $A_{i}$, since the kinetics of topochemical reactions (as well as during drying) are significantly affected by geometric factors of shape, crystal structure, interface and relative phase surface velocity, and others [2]. Therefore, without taking these factors into account, the results of determining the value of $A_{i}$, for example, obtained using thermal analysis devices [19, 20] cannot be transferred to mathematical models of industrial processes. In its physical meaning, the coefficient $A_{i}$ represents the maximum value of the drying rate at a very high temperature, when neither the activation energy nor the moisture content influence on the speed process $\left(f(\alpha)=1 ; \exp \left(-\frac{E}{R T}\right)=1\right)$. In this regard, the determination of the value of $A_{i}$ is a separate task of scientific research. In the simplest case, information on the coefficient $A_{i}$ can be obtained experimentally, as shown in [5].

Thus, the development of a mathematical model of drying based on the laws of chemical kinetics should be performed in the following sequence:

1) The fractional composition of moisture in the product is investigated and the boundaries of water fractions are determined.

2) The activation energy of each water fraction is determined.

3) Based on information about the forms of moisture coupling in the material, the sequence of removal of water fractions (sequential or parallel) and the type of kinetic equation for periods of constant and decreasing drying speed are determined.

4) The type of function of the degree of water fractions transformation is determined.

5) We conduct a theoretical or experimental study to determine the value of the pre-exponential multiplier of the kinetic equation.

6) The resulting drying model is examined for suitability for practical use. 


\section{CONCLUSION}

Drying process can be considered from the standpoint of physical chemistry as a quasitopochemical heterogeneous reaction. Mathematical modeling of the process can be based on application of laws of chemical kinetics.

The type of such a quasitopochemical heterogeneous reaction (sequential or parallel removal of water fractions) is determined on the basis of studying the forms and energy of water binding to the dry part of the product. The boundaries of aqueous fractions, which differ in the binding energy of moisture, can be reliably determined by nuclear magnetic resonance and thermal analysis.

The value of activation energy can be defined as the sum of the binding energy of moisture with the dry part of the product, the latent heat of vaporization and the energy needed to move water vapor through the capillaries in the dry layer of the product. Activation energy is a function of the temperature and moisture content of the product. The value of the activation energy can be determined by the thermal analysis method.

To establish the dependence of pre-exponential coefficients of the Arrhenius equation on the geometric factors of the form, the relative motion of the phases and other factors, additional research is needed.

The considered methodology is applicable primarily to convective drying of dispersed products with an active hydrodynamic regime, when the evaporation zone can be considered from the point of view of chemical kinetics as a kinetic region for small particle sizes. Therefore, as the next research tasks, it is advisable to justify the type of kinetic equation and develop a simulation algorithm for other drying methods: drying with heat input by high frequency currents, infrared rays, etc. Further improvement of mathematical models of drying is possible on the basis of a detailed study of the transformation function of water fractions and the preexponential coefficients of the Arrhenius equation.

The implementation of the physicochemical approach to drying process modeling allows one to obtain simple and reliable mathematical models of drying kinetics for engineering practice and to avoid errors that the authors note in previously published works.

\section{Practical Implementation of SCIENTIFIC ReSEARCH RESULTS}

For the practical implementation and development of the idea of applying the laws of chemical kinetics to mathematical modeling of drying processes, various classification groups of products should be studied according to the indicated methodology: colloidal, capillary-porous, and others. Also it is also needed to establish the kinetic laws of drying explicitly for each group of products and, on this basis, to develop algorithms for computer technology for controlling production processes.

\section{ACKNOWLEDGMENT}

The work was carried out with the financial support of the Russian Foundation for Basic Research in the framework of grant No. 19-016-00049 "Development of a software system for automated calculation of the composition of flour mixtures of functional and dietary nutrition".

\section{REFERENCES}

[1] Patent of the Russian Federation No. 2444689, PMK F26B 25/22, C1. A method for automatic control of the drying process of food products in a belt dryer using convective and microwave energy supply / S.T. Antipov, D.A. Kazartsev, A.V. Zhuravlev, T.V. Kalinina, I.S. Yurova, A.B. Emelyanov. - Application: No. 2010135851/06; 08/26/2010, publ. 03/10/2012. Bull. Number 7.

[2] Patent of the Russian Federation No. 2547345, PMK F26B 25/22, C1. A method for automatic control of the drying process of dispersed materials in a swirling coolant flow with microwave energy supply / D.A. Kazartsev, S.T. Antipov, A.V. Zhuravlev, D.A. Nesterov, A.V. BoRodkina, S.A. Vinichenko. - Application: No. 2013156470/06; 12/19/2013, publ. 04/10/2015. Bull. Number 10.

[3] Sazhin, B.S. Scientific principles of drying technology / B.S. Sazhin, V.B. Sazhin. - M .: Nauka, 1997 .- 448 p.

[4] Rudobashta S. P. Using the theoretical propositions of academician A.V. Lykov in modern models of heat and mass transfer during drying / Actual problems of drying and heat-humidity processing of materials in various industries and agro-industrial complex / / collection of scientific articles of the First international Lykov scientific readings. Kursk, 2015. P. $21-28$.

[5] Fedosov S. V. Heat and mass transfer in technological processes of the construction industry. Ivanovo: IPK "Pressto". 2010. - 364 p.

[6] Rudobashta S. P. New Russian studies in the field of drying and thermal-moisture processes / / Trudy 3 mezhdunarod. science. practical. Conf. "Modern energy-saving thermal technologies (Drying and thermal processes SETT-2008)". Vol. 1. Moscow, 2008. - Pp. 5-18.

[7] Antipov, S.T. Development of a mathematical model of the drying process of blackcurrant fruits in a vacuum apparatus with microwave energy supply / S.T. Antipov, D.A. Kazartsev, A.V. Zhuravlev, S.A. Vinichenko // Bulletin of the Voronezh State University of Engineering Technologies - Voronezh: Publishing House of the Voronezh State. University of Technology, 2014. - P. 7-12.

[8] Yurova, I.S., Heat and mass transfer during drying of milk thistle seeds in a vortex chamber with microwave energy supply: Monograph / I.S. Yurova, I.T. Kretov, A.V. Zhuravlev, D.A. Kazartsev. - Voronezh: Publishing house of the Voronezh State University of Engineering Technology, 2012. - $192 \mathrm{p}$.

[9] CFD simulation of spray dryers / M.W. Woo, L.X. Huang, A.S. Mujumdar, W.R.W. Daud ; Ed.M.W. Woo, A.S. Mujumdar, W.R.W. Daud. - Singapore, 2010. - V. 1. - P. 1 - 36. - ISBN 978-981- 08-62701.

[10] Yoon, S.S. Lagrangian-based stochastic dilute spray modelling for drying applications / S.S. Yoon ; Ed.M.W. Woo, A.S. Mujumdar, W.R.W. Daud. - Singapore, 2010. - V. 1. - P. 77 - 112. - ISBN 978981- 08-6270-1.

[11] Julklang, W. Analysis of Slurry Drying in a Spray Dryer / W. Julklang, B. Golman // International Journal of Engineering and Technology (IJET). - Dec 2013 - Jan 2014. - V. 5. - N. 6. - P. 5178 - 5189. DOI:10.1007/s11671-010-9793-9.

[12] Handbook of Industrial Drying / Fourth Edition Edited by Arun S. Mujumdar // CRC Press Taylor \& Francis Group. - 2015. - P. 1334.

[13] Dornyak O. R. Modern problems of mathematical modeling of thermalhumidity processing of materials / collection of scientific articles of the First international Lykov scientific readings. Kursk. P. 36 - 42.

[14] Dyachenko, A.N. Chemical kinetics of heterogeneous processes: a training manual / / A.N. Dyachenko, V.V. Shagalov // Tomsk Polytechnic University. - Tomsk: Publishing House of Tomsk Polytechnic University, 2014. - 102 p.

[15] Arapov, V.M. Improving the drying of casein Spec. 05.18 .12 - Processes and Food Production Equipment / Diss. for Ph.D. Moscow, 1985 .- 146 p.

[16] Drannikov, A.V. Study of the drying process of beet pulp with superheated steam Specialty: 05.18.12-Processes and food production apparatus / Diss. for Ph.D. Voronezh - 2003. - 164 p. 
[17] Antipov, S.T., Heat and mass transfer during drying of coriander seeds in an apparatus with microwave energy supply: Monograph / S.T. Antipov, D.A. Kazartsev. - Voronezh: Publishing house of the Voronezh state. Technological Acad., 2007. - 142 p.

[18] Mamontov M.V. Development and research of drying finely chopped carrots during its complex processing/ Specialty: 05.18.12-Processes and food production apparatus / Diss. for Ph.D. Voronezh - 2009. - 184 p.

[19] Ermochenkov M.G. Kinetic parameters of the wood drying process // Lesn. journal 2017. No. 6. P. 114-125. (Izv. Higher education. Institutions).

[20] Kuvik T.Ye. Kinetics of evaporation of bound moisture and wood destruction during thermal modification / Diss. abstract for Ph.D. Moscow - 2013. - $20 \mathrm{p}$.

[21] Ermochenkov M.G. Internal sources of mass during wood drying / M.G. Ermochenkov // Materials of the VI B.N. Ugolev international symposium, dedicated to the 50th anniversary of the Regional Coordinating Council on Contemporary Problems of Wood Science (Krasnoyarsk, September 10-16, 2018) - Novosibirsk: Publishing House of the SB RAS, 2018.- P. 80 - 82.

[22] Kholmansky A.S., Tilov A.Z., Sorokina E.Yu. Physicochemical modeling of the drying process of vegetables and fruits / A.S Kholmansky, A.Z. Tilov, E.Yu. Sorokina // Modern problems of science and education. - 2012. - No. 5; URL: http://www.science-education.ru.
[23] Patent of the Russian Federation No. 2204822, PMK G01N 24/00, C2. A method for determining the amount of monomolecular - adsorption and polymolecular - adsorption moisture / I.T. Kretov, V.M. Arapov, S.V. Shakhov. - Application: No. 2001123030/28, 15. 08. 2001; publ. 20. 05. 2003 Bull. No. 14.

[24] Patent of the Russian Federation No. 2296974, PMK G01N 15/00, C1. A method for determining the fractional composition of moisture in materials / V.M. Arapov, M.V. Mamontov, M.V. Arapov. - Application: No. 2005122966/28, 19. 07. 2005; publ. 10.04. 2007 Bul. No. 1.

[25] Patent of the Russian Federation No. 2312328, PMK G01N 25/56, C2. A method for determining the amount of aqueous fractions that differ in the binding energy of moisture with a substance / V.M. Arapov, S.V. Shakhov, M.V. Arapov, S.V. Buturlin. - Application: No. 2006100224/13, 10. 01. 2006; publ. 10. 12. 2007 Bull. Number 34.

[26] Patent of the Russian Federation No. 2230311, PMK G01N 25/56, C1 Method for determining the bond strength of moisture with a substance / V.M. Arapov, D.A. Kazartsev, M.V. Arapov. - Application: No. 2003103805/28, 10. 02. 2003; publ. 10.06. 2004 Bul. No. 16.

[27] Patent of the Russian Federation No. 2292018, PMK G01J 5/56, C1. A method for determining the binding energy of moisture with a substance / V. M. Arapov, M.V. Mamontov, M.V. Arapov. - Application: No. 2005121839/28; 07/11/2005, publ. 20. 01. 2007 Bull. No. 2. 